\title{
Subjective evaluation of the frequency of salty food intake and its relationship to urinary sodium excretion and blood pressure in a middle-aged population
}

\author{
Toshiaki Otsuka $\cdot$ Katsuhito Kato $\cdot$ Chikao Ibuki • \\ Eitaro Kodani · Yoshiki Kusama · Tomoyuki Kawada
}

Received: 10 August 2012/Accepted: 19 November 2012/Published online: 12 December 2012

(C) The Japanese Society for Hygiene 2012

\begin{abstract}
Objectives The aim of this study was to examine the relationship between subjective evaluations of the frequency of salty food intake and both urinary sodium excretion and blood pressure (BP) in a middle-aged population.

Methods Urinary sodium and creatinine concentrations in a spot sample and brachial BP were measured in 419 subjects (mean age $37 \pm 9$ years, 385 men) during a health examination at a company in Kanagawa, Japan, 2011. Twenty-four-hour urinary sodium excretion was estimated from sodium and creatinine concentrations in the spot sample. The subjects were asked about the frequency of salty food intake on a self-reported questionnaire, choosing their answer from 'seldom', 'sometimes', or 'always' based on their own subjective evaluation. The amount of daily salt intake was converted from 24-h urinary sodium excretion.

Results The prevalence of high salt intake, defined as greater than the daily average salt intake of Japanese according to the National Health and Nutrition Survey in 2010, gradually increased from the 'seldom' to the
\end{abstract}

T. Otsuka $(\bowtie) \cdot$ K. Kato · T. Kawada

Department of Hygiene and Public Health,

Nippon Medical School, 1-1-5 Sendagi, Bunkyo-ku,

Tokyo 113-8602, Japan

e-mail: otsuka@nms.ac.jp

C. Ibuki

Cardiovascular Center, Nippon Medical School Chiba-Hokusoh

Hospital, Inzai, Chiba, Japan

E. Kodani · Y. Kusama

Department of Internal Medicine and Cardiology,

Nippon Medical School Tama-Nagayama Hospital,

Tama, Tokyo, Japan 'sometimes', and then 'always' categories (10.3, 13.4, and $24.0 \%$, respectively; $P=0.013$ for trend). The 24 -h urinary sodium excretion among these groups also tended to be different; however, the differences did not reach a significantly significant level $(145 \pm 41,152 \pm 34$, and $160 \pm 40 \mathrm{mEq} /$ day, respectively; $P=0.076$ ). Both systolic and diastolic BP were similar among the groups.

Conclusions Although some of our findings raise the possibility of a relationship between a subjective evaluation of the frequency of salty food intake and urinary sodium excretion, further studies are needed to confirm the relationship. In contrast, no relationship with BP was found.

Keywords Blood pressure - Hypertension .

Questionnaire $\cdot$ Salt intake · Urinary sodium excretion

\section{Introduction}

The association between excess salt intake and an elevation in blood pressure (BP) has been shown in a number of epidemiologic and clinical studies [1-3]. It is therefore crucial to identify subjects with excess salt intake for the prevention of future BP elevation. There are several objective and reliable methods for evaluating salt intake, such as the measurement of urinary sodium excretion, a record of consumed foods, and weighing method [4]. However, actually carrying out these procedures in the field of general health examination is difficult because such methods are complicated and time-consuming. A previous study showed an association between self-reported salt preference and daily salt intake as evaluated by urinary sodium excretion [5]. These findings suggest the potential usefulness of subjectively evaluated information on salty foods intake for estimating the amount of actual salt intake. 
However, that study could not show a difference in the BP between salt preference and non-salt preference subjects, even though excess salt intake may increase BP.

To further elucidate the usefulness of self-reported information on salt intake, we have examined the relationship between the subjectively evaluated frequency of salty food intake and both urinary sodium excretion and BP in a middle-aged Japanese population.

\section{Methods}

\section{Study population}

This study was conducted during an annual health examination at a semiconductor manufacturing plant in Kanagawa, Japan, in 2011. A total of 499 individuals underwent the health examination, and 438 of these gave informed consent to participate in the present study. Of these 438 individuals, 19 were excluded from the study because they were taking antihypertensive medications. We initially intended to exclude those individuals with a history or presence of cardiovascular disease, but no such individuals were present among the 438 individuals screened. The final study cohort comprised 419 individuals (mean age $37 \pm 9$ years, 385 males). This study was approved by the Ethics Committee of Nippon Medical School, and all participants gave written informed consent.

\section{Measurement of variables}

All participants underwent anthropometric and BP measurements and blood and urine sampling. Body weight and height were measured, and the body mass index (BMI) was calculated as the weight $(\mathrm{kg})$ divided by the square of the height $\left(\mathrm{m}^{2}\right)$. Obesity was defined as a BMI of $\geq 25.0 \mathrm{~kg} / \mathrm{m}^{2}$. The right brachial BP was measured with the subject in a seated position using a mercury sphygmomanometer. Hypertension was defined as a systolic BP of $\geq 140 \mathrm{mmHg}$ or a diastolic BP of $\geq 90 \mathrm{mmHg}$. Blood samples were obtained from the antecubital vein after overnight fasting. The serum low-density and high-density lipoprotein cholesterol, triglycerides, plasma glucose levels, and HbA1c were then measured. Dyslipidemia was defined as a lowdensity lipoprotein-cholesterol level of $\geq 140 \mathrm{mg} / \mathrm{dl}$, a high-density lipoprotein-cholesterol level of $<40 \mathrm{mg} / \mathrm{dl}$, a triglyceride level of $\geq 150 \mathrm{mg} / \mathrm{dl}$, or the use of lipid-lowering medications. Impaired fasting glucose/diabetes mellitus was defined as a fasting plasma glucose level of $\geq 110 \mathrm{mg} / \mathrm{dl}$, HbA1c of $\geq 6.5 \%$, or the use of glucoselowering medications.

Urinary sodium and creatinine concentrations were measured using a spot sample. The 24-h urinary sodium excretion was estimated using the following equation, as presented by Tanaka et al. [6] and recommended by the Japanese Society of Hypertension [7]: 24-h urinary sodium excretion (mEq/ day) $=21.98 \times\left[(\mathrm{NaS} / \mathrm{CrS}) \times \mathrm{Pr} \mathrm{UCr}_{24}\right]^{0.392}$, where $\mathrm{NaS}$ indicates the sodium concentration in the spot urine sample $(\mathrm{mEq} / \mathrm{l}), \mathrm{CrS}$ indicates the creatinine concentration in the spot urine sample $(\mathrm{mg} / \mathrm{l})$, and $\mathrm{Pr} \mathrm{UCr}_{24}$ indicates the estimated 24-h urinary creatinine excretion (mg/day) calculated as $-2.04 \times$ age $($ years $)+14.89 \times$ weight $(\mathrm{kg})+16.14 \times$ height $(\mathrm{cm})-2,244.45$. Because most of the salt (sodium) ingested is thought to be excreted in urine, we regarded the 24-h urinary sodium excretion as a reflection of daily salt intake, in accordance with the recommendations of the Japanese Society of Hypertension [4]. The daily salt intake (g/day) was converted from the 24-h urinary sodium excretion (mEq/ day) multiplied by 0.0585 [4]. We adopted two definitions of high salt intake. Definition 1 defined high salt intake as greater than the average daily salt intake of Japanese ( $\geq 11.5 \mathrm{~g} /$ day for men and $\geq 9.9 \mathrm{~g} /$ day for women) according to the National Health and Nutrition Survey in 2010 [8]. Definition 2 defined high salt intake as greater than the recommended amount of daily salt intake ( $\geq 9.0 \mathrm{~g} /$ day for men and $\geq 7.5 \mathrm{~g} /$ day for women) according to the Dietary Reference Intakes for Japanese in 2010 presented by the Ministry of Health, Labour and Welfare [9].

A self-reported questionnaire was used to collect the information on the subjects' smoking status, alcohol intake, and frequency of salty food intake. Excess alcohol intake was defined as an ethanol intake of $\geq 300 \mathrm{~g} /$ week [10]. The subjects were also asked "How often do you eat salty foods?". The possible answers from which they could chose were 'seldom', 'sometimes', or 'always', and the answers were taken to be a subjective assessment. To answer the question, the subjects were required to decide upon two issues based on their feelings: (1) whether the consumed foods were actually salty or not; (2) the frequency at which they consumed salty foods.

Statistical analysis

All statistical analyses were performed using SPSS software (ver. 19.0.0; IBM Corp, Somers, NY) or MedCalc software (ver 10.1.4; MedCalc Software, Mariakerke, Belgium). Continuous and categorical variables were expressed as the mean \pm standard deviation (SD) and the percentage of the total, respectively. The differences in the variables among the 'seldom,' 'sometimes,' and 'always' groups were compared by an analysis of variance or the chi-square test (and chi-square test for trend, if needed), as appropriate. The 24-h urinary sodium excretion and BP among the groups were also compared using an analysis of covariance; variables that showed a $P$ value of $<0.1$ by an analysis of variance or the chi-square test were used as 
covariates. Systolic and diastolic BP were further compared among the groups with the inclusion of other potential confounding factors in the models as covariates. All statistical tests were two-sided, and a $P$ value of $<0.05$ was considered to be significant.

\section{Results}

There were significant differences in age and the prevalence of obesity among the 'seldom' $(n=39)$, 'sometimes' $(n=284)$, and 'always' $(n=96)$ groups (Table 1). The prevalence of male sex tended to be different among the groups, but the other characteristics were similar.

Overall, the 24-h urinary sodium excretion (daily salt intake) was $153 \pm 36 \mathrm{mEq} /$ day $(9.0 \pm 2.1 \mathrm{~g} /$ day $)$, and the systolic and diastolic BP were $122 \pm 10$ and $71 \pm 9 \mathrm{mmHg}$, respectively.

The 24-h urinary sodium excretion (daily salt intake) among the 'seldom,' 'sometimes,' and 'always' groups are shown in Table 2. The prevalence of high salt intake according to Definition 1 gradually increased across the groups ( $P=0.013$ for trend). When Definition 2 was used, the prevalence of high salt intake across the groups also gradually increased; however, it did not reach a statistically significant level $(P=0.096$ for trend). Similarly, the $24-\mathrm{h}$ urinary sodium excretion gradually increased across the groups, but the difference did not reach a statistically significant level $(P=0.075)$. However, after adjusting for age, sex, and obesity, which were the variables showing a

Table 1 Characteristics of the study participants according to the subjectively evaluated frequency of salty food intake

\begin{tabular}{lllll}
\hline $\begin{array}{l}\text { Patient } \\
\text { characteristics }\end{array}$ & \multicolumn{2}{l}{ Frequency of salty foods intake } & $P$ value \\
\cline { 2 - 4 } & $\begin{array}{l}\text { Seldom } \\
(n=39)\end{array}$ & $\begin{array}{l}\text { Sometimes } \\
(n=284)\end{array}$ & $\begin{array}{l}\text { Always } \\
(n=96)\end{array}$ & \\
\hline Age (years) & $39 \pm 8$ & $38 \pm 9$ & $35 \pm 8$ & $0.001^{\mathrm{a}}$ \\
Male sex & 84.6 & 91.5 & 95.8 & $0.090^{\mathrm{b}}$ \\
Obesity & 7.7 & 25.4 & 20.8 & $0.042^{\mathrm{b}}$ \\
Hypertension & 0.0 & 5.6 & 3.1 & $0.21^{\mathrm{b}}$ \\
Dyslipidemia & 30.8 & 36.6 & 35.4 & $0.77^{\mathrm{b}}$ \\
IFG/DM & 5.1 & 4.6 & 5.2 & $0.96^{\mathrm{b}}$ \\
Current & 25.6 & 32.7 & 34.4 & $0.61^{\mathrm{b}}$ \\
$\quad$ smoking & & & & \\
Excess & 0.0 & 4.2 & 5.2 & $0.37^{\mathrm{b}}$ \\
$\begin{array}{l}\text { alcohol } \\
\text { intake }\end{array}$ & & & & \\
\hline
\end{tabular}

IFG/DM Impaired fasting glucose/diabetes mellitus

Data are presented as the percentage of participants, except for age, which is presented as the mean \pm standard deviation (SD)

a Analysis of variance

b Chi-square test
$P$ value of $<0.1$ by an analysis of variance or the chi-square test, the 24-h urinary sodium excretion showed a significant difference among groups $(P=0.049)$.

The systolic and diastolic BP were similar among the groups, even after adjusting for age, sex, and obesity (Table 2). These findings remained insignificant after further adjusting for multiple potential confounders, including dyslipidemia, impaired fasting glucose/diabetes mellitus, current smoking, and the categorized amount of weekly ethanol intake $(0, \leq 99,100-199,200-299$, and $\geq 300 \mathrm{~g} /$ week) (data not shown). When the BMI was used as a continuous variable instead of obesity in the analysis, the insignificance of the results remained (data not shown).

\section{Discussion}

In the present study, the prevalence of high salt intake, defined as more than average daily salt intake of Japanese according to the National Health and Nutrition Survey in 2010 [8], gradually increased across the 'seldom,' 'sometimes,' and 'always' groups. In contrast, the 24-h urinary sodium excretion did not reach a statistically significant difference among the groups, which appears to be in conflict with previous observations $[5,11]$. However, after adjusting for age and the prevalence of obesity and male sex, the 24-h urinary sodium excretion showed significant differences among the groups. Taken together, although some of our findings raise the possibility of a relationship between a subjective evaluation of the frequency of salty food intake and urinary sodium excretion, further studies are needed to confirm the relationship.

Similar to previously reported results [5], the present study failed to show a relationship between the subjective evaluation of salty food intake and BP. One possible reason for this lack of relationship being identified may be the small differences in daily salt intake among the three groups (the maximum differences in the mean value of $0.8-0.9 \mathrm{~g} /$ day). This small difference may have resulted in a difference in systolic BP of $1 \mathrm{mmHg}$ among the groups, which is clearly not a statistically significant difference. These results are supported by the findings of a previously reported meta-analysis that indicated a decrease in systolic $\mathrm{BP}$ of approximately $1 \mathrm{mmHg}$ with a decrease in daily salt intake of $1 \mathrm{~g} /$ day [3]. Another possible reason is that the present study is a cross-sectional investigation. Therefore, subjects with a high BP may already be attempting to reduce their salt intake.

There are limitations to the present study. First, the 24-h pooled urine was not used to obtain the daily urinary sodium excretion; alternatively, it was estimated from a spot urine sample. Indeed, only a moderate correlation has 
Table 2 Comparison of 24-h urinary sodium excretion and blood pressure among the groups according to the subjectively evaluated frequency of salty food intake

\begin{tabular}{|c|c|c|c|c|}
\hline \multirow[t]{2}{*}{ Parameters } & \multicolumn{3}{|c|}{ Frequency of salty food intake } & \multirow[t]{2}{*}{$P$ value } \\
\hline & Seldom & Sometimes & Always & \\
\hline \multicolumn{5}{|l|}{ High salt intake $(\%)$} \\
\hline Definition $1^{\mathrm{a}}$ & 10.3 & 13.4 & 24.0 & $\begin{array}{l}0.030^{\mathrm{e}} \\
0.013^{\mathrm{f}}\end{array}$ \\
\hline Definition $2^{\mathrm{b}}$ & 41.0 & 47.2 & 55.2 & $\begin{array}{l}0.25^{\mathrm{e}} \\
0.096^{\mathrm{f}}\end{array}$ \\
\hline \multicolumn{5}{|l|}{ 24-h urinary sodium excretion } \\
\hline $\begin{array}{l}\text { mEq/day } \\
\text { g/day }\end{array}$ & $\begin{array}{l}145 \pm 41 \\
8.5 \pm 2.4\end{array}$ & $\begin{array}{l}152 \pm 34 \\
8.9 \pm 2.0\end{array}$ & $\begin{array}{l}160 \pm 40 \\
9.4 \pm 2.4\end{array}$ & $0.075^{\mathrm{g}}$ \\
\hline \multicolumn{5}{|c|}{ Adjusted $^{\mathrm{d}} 24$-h urinary sodium excretion } \\
\hline $\begin{array}{l}\text { mEq/day } \\
\text { g/day }\end{array}$ & $\begin{array}{l}147 \pm 36 \\
8.6 \pm 2.1\end{array}$ & $\begin{array}{l}152 \pm 36 \\
8.9 \pm 2.1\end{array}$ & $\begin{array}{l}161 \pm 36 \\
9.4 \pm 2.1\end{array}$ & $0.049^{\mathrm{h}}$ \\
\hline Systolic BP (mmHg) & $121 \pm 9$ & $122 \pm 11$ & $122 \pm 11$ & $0.70^{\mathrm{g}}$ \\
\hline Diastolic BP (mmHg) & $69 \pm 6$ & $71 \pm 9$ & $70 \pm 9$ & $0.21^{\mathrm{g}}$ \\
\hline Adjusted $^{\mathrm{d}}$ systolic BP (mmHg) & $122 \pm 10$ & $122 \pm 10$ & $123 \pm 10$ & $0.83^{\mathrm{h}}$ \\
\hline Adjusted $^{\mathrm{d}}$ diastolic $\mathrm{BP}(\mathrm{mmHg})$ & $69 \pm 8$ & $71 \pm 8$ & $71 \pm 8$ & $0.34^{\mathrm{h}}$ \\
\hline
\end{tabular}

$B P$ Blood pressure

Data are presented at the mean \pm SD unless indicated otherwise

${ }^{\text {a }}$ Defined as a daily salt intake of more than average daily salt intake of Japanese ( $\geq 11.5 \mathrm{~g} /$ day for men and $\geq 9.9 \mathrm{~g} / \mathrm{day}$ for women) according to the National Health and Nutrition Survey in 2010

b Defined as a daily salt intake of more than the recommended amount of daily salt intake ( $\geq 9.0 \mathrm{~g} /$ day for men and $\geq 7.5 \mathrm{~g} / \mathrm{day}$ for women) according to the Dietary Reference Intakes for Japanese in 2010 presented by the Ministry of Health, Labour and Welfare

${ }^{\mathrm{c}}$ Converted values to daily salt intake (g/day) from 24-h urinary sodium excretion (mEq/day), multiplied by 0.0585

d Adjusted for age, sex, and obesity

e Chi-square test

f Chi-square test for trend

$\mathrm{g}$ Analysis of variance

h Analysis of covariance

been reported between the actual measured 24-h urinary sodium excretion and that estimated from a spot sample ( $r=0.54$ ), and in that study the estimated values from spot urine samples were lower than the actual measured values from 24-h pooled urine [6]. In addition, all ingested salt is not necessarily excreted through urine only. Therefore, caution is needed when interpreting our present results; for example, the prevalence of high salt intake presented here may be lower than the true prevalence. However, it is difficult to fully carry out 24-h urine collection in real occupational settings as well as during general health examinations. Second, the study participants included only middle-aged Japanese workers at a single manufacturer. Therefore, it is unclear whether the present results can be extrapolated to other populations, including the elderly and other ethnic groups.

In conclusion, some of the present findings suggest that subjective evaluations of the frequency of salty food intake were related to urinary sodium excretion. These findings raise the possibility that such information is useful for identifying subjects with high salt intake in a middle-aged Japanese population. However, all of our results do not necessarily support this relationship; therefore, further studies are needed to confirm such a relationship. In contrast, the relationship between the subjective evaluation of salty foods intake and BP was not found in our study. Further studies are also needed to clarify this relationship.

Acknowledgments The authors wish to thank Chiharu Komiya and Yuka Sugimori for their technical assistance in collecting the data.

Conflict of interest The authors declare that there are no conflicts of interest.

\section{References}

1. Luft FC, Rankin LI, Bloch R, Weyman AE, Willis LR, Murray $\mathrm{RH}$, et al. Cardiovascular and humoral responses to extremes of sodium intake in normal black and white men. Circulation. 1979;60:697-706. 
2. Intersalt Cooperative Research Group. Intersalt: an international study of electrolyte excretion and blood pressure. Results for 24 hour urinary sodium and potassium excretion. $\mathrm{Br}$ Med $\mathrm{J}$. 1988;297:319-28.

3. He FJ, MacGregor GA. Effect of modest salt reduction on blood pressure: a meta-analysis of randomized trials. Implications for public health. J Hum Hypertens. 2002;16:761-70.

4. Kawano Y, Tsuchihashi T, Matsuura H, Ando K, Fujita T, Ueshima H. Report of the Working Group for Dietary Salt Reduction of the Japanese Society of Hypertension: (2) Assessment of salt intake in the management of hypertension. Hypertens Res. 2007;30:887-93.

5. Hashimoto T, Yagami F, Owada M, Sugawara T, Kawamura M. Salt preference according to a questionnaire vs. dietary salt intake estimated by a spot urine method in participants at a health checkup center. Intern Med. 2008;47:399-403.

6. Tanaka T, Okamura T, Miura K, Kadowaki T, Ueshima H, Nakagawa $\mathrm{H}$, et al. A simple method to estimate populational 24-h urinary sodium and potassium excretion using a casual urine specimen. J Hum Hypertens. 2002;16:97-103.
7. Ogihara T, Kikuchi K, Matsuoka H, Fujita T, Higaki J, Horiuchi $\mathrm{M}$, et al. The Japanese Society of Hypertension Guidelines for the Management of Hypertension (JSH 2009). Hypertens Res. 2009;32:3-107.

8. Ministry of Health, Labour and Welfare. 2010 National Health and Nutrition Survey (in Japanese). Available at: http://www. mhlw.go.jp/bunya/kenkou/eiyou/dl/h22-houkoku-01.pdf. Accessed 29 Oct 2012

9. Ministry of Health, Labour and Welfare. Dietary Reference Intakes for Japanese in 2010. Available at: http://www0.nih.go. jp/eiken/info/pdf/dris2010en2.pdf. Accessed: 29 Oct 2012

10. Tsugane S, Fahey MT, Sasaki S, Baba S. Alcohol consumption and all-cause and cancer mortality among middle-aged Japanese men: seven-year follow-up of the JPHC study Cohort I. Japan Public Health Center. Am J Epidemiol. 1999;150:1201-7.

11. Ohta Y, Tsuchihashi T, Ueno M, Kajioka T, Onaka U, Tominaga $\mathrm{M}$, et al. Relationship between the awareness of salt restriction and the actual salt intake in hypertensive patients. Hypertens Res. 2004;27:243-6. 